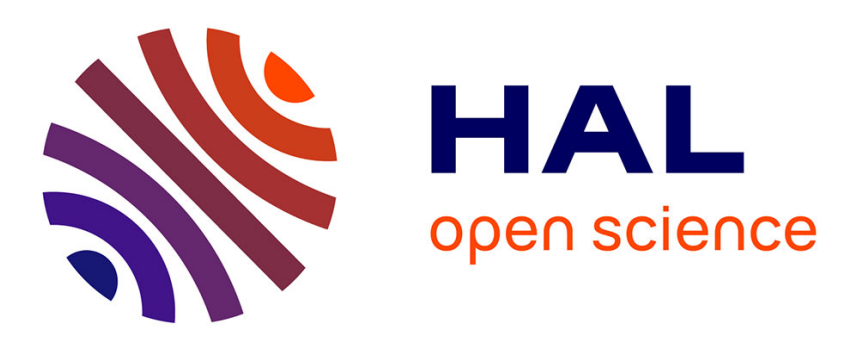

\title{
Adhesion of lubricant on aluminium through adsorption of additive head-groups on $\gamma$-alumina: A DFT study
}

\author{
Sarah Blanck, Sophie Loehle, Stephan N. Steinmann, Carine Michel
}

\section{To cite this version:}

Sarah Blanck, Sophie Loehle, Stephan N. Steinmann, Carine Michel. Adhesion of lubricant on aluminium through adsorption of additive head-groups on $\gamma$-alumina: A DFT study. Tribology International, 2020, 145, pp.106140. 10.1016/j.triboint.2019.106140 . hal-02917727

\section{HAL Id: hal-02917727 \\ https://hal.science/hal-02917727}

Submitted on 19 Aug 2020

HAL is a multi-disciplinary open access archive for the deposit and dissemination of scientific research documents, whether they are published or not. The documents may come from teaching and research institutions in France or abroad, or from public or private research centers.
L'archive ouverte pluridisciplinaire $\mathbf{H A L}$, est destinée au dépôt et à la diffusion de documents scientifiques de niveau recherche, publiés ou non, émanant des établissements d'enseignement et de recherche français ou étrangers, des laboratoires publics ou privés. 


\section{Adhesion of lubricant on aluminium through adsorption of}

\section{additive head-groups on $\gamma$-alumina: a DFT study}

Sarah Blanck ${ }^{\mathrm{a}, \mathrm{b}}$, Sophie Loehléb ${ }^{\mathrm{b}}$, Stephan N. Steinmann ${ }^{\mathrm{a}}$, Carine Michel ${ }^{\mathrm{a}, *}$

a. Univ Lyon, ENS de Lyon, CNRS UMR 5182, Université Claude Bernard Lyon 1, Laboratoire de Chimie, F69342, Lyon, France

b. Centre de Recherche Total de Solaize, Chemin du Canal - BP 2269360 Solaize, France

* carine.michel@ens-lyon.fr

\section{Abstract}

To improve lubricant formulation, a better understanding of the wettability properties and hence of the interactions between the surface and the lubricant additives is a key parameter. Herein, we use density functional theory calculations to characterize 32 typical head groups, classified into four categories: protic, aprotic, aromatic and phosphorus-containing molecules. By comparing their adsorption energies on $\gamma$-alumina, used as a surface model of aluminium sheets, and their solvation energies in a model lubricant base oil, we found that the solvation energy was not a discriminant parameter while the adsorption energy was critical. Phosphates and carboxylic acids are the most strongly adsorbed, and thus more likely to yield to improved wettability properties of the lubricant through film formation. 


\section{Introduction}

Lubricants are widely used formulated products, with a global market size valued over USD 125 billion in 2018. The major applications occur in the automotive, the marine, and the industrial (metal working and textile) sectors. They are usually composed of a base oil which can be made of either mineral, vegetal or synthetic oils depending on the usage [1], and a package of additives used to enhance the properties of interest of the base oil or add new properties to the lubricant, generally leading to a rather complex composition. A wide range of additives for various purposes can be found in lubricants. Empirically, they are classified into different groups depending on their function in the lubricant, e.g., surfactants, friction modifiers, anti-wear and extreme pressure additives and corrosion inhibitors [2-4]. In this study, we focus on lubricants to be used in metalworking of aluminium sheets, aluminium being for instance more and more used as a cost-effective alternative to steel in the automotive industry where weight reduction is key to both fuel-operated and electric vehicles. In this field, friction modifiers are the most studied additives, as they directly influence the tribological properties of lubricants [5-7]. On the contrary, only few studies have discussed the wettability properties of lubricants and more generally the influence of additives on the wetting of a given surface $[8,9]$, which is the main focus of the present study.

Even if lubricants are used for centuries [2], their development is still ongoing and the lubricants composition is constantly evolving. Indeed, changes in formulations are triggered mostly by modifications of legislations and by the constant search for better performances. The changes imposed by more stringent legislation can lead to significant effects on the behavior of the lubricant towards the surface, e.g., it can deteriorate the wettability property of the lubricant, i.e., its ability to quickly spread over the surface to achieve its optimal tribological performance $[10,11]$. Therefore, it is important to be 
able to understand and to predict the effects of the different components of the lubricant and their link with the wettability properties.

Indeed, surface engineering to achieve the desired wettability is an active field of research. These modifications can be obtained by either chemical of physical processes [12-14] and can even lead to superhydrophobic or superhydrophilic properties [15-18]. However, in the case of lubrication, the surface is often predetermined and cannot be cost-efficiently modified before application of the lubricant to increase its wettability. Hence, the lubricant formulations need to be adapted to get an acceptable wettability.

A large number of experimental methods have been developed to measure the contact angle of a liquid on a surface [19] but in some case, contact angles are not sufficient to describe the difference in wetting observed between two liquids $[20,21]$ and other techniques are needed to be able to predict the difference in the wettability properties. Theoretical models have been developed by Blake et al. to describe the dynamics of wetting of a liquid drop on a surface $[22,23]$. They showed that solid-liquid interactions affect the spreading of the liquid on the surface in two opposite directions: on the one hand, the interaction between the liquid and the solid constitutes the driving force for spreading and, on the other hand, too strong interactions result in a resistance to wetting. Therefore, very much like a volcano curve in catalysis [24], a system dependent optimal value of solid-liquid interactions, corresponding to the maximal wetting of surface, should be aimed at. Furthermore, to affect the wettability, the additives should have the tendency to go to the solid-liquid interface rather than to stay in the liquid bulk, i.e., their solvation free energy should not be more important than the adsorption free energy. 
Based on these considerations, we set out to theoretically compare the adsorption and the solvation energies of different types of additive head groups, aiming to classify them according to their expected influence on the spreading of the lubricant on the considered surface. DFT is the method of choice for theoretically determining the adsorption free energy of additives on surfaces. In contrast to force fields, this first principles method is applicable to any head group imaginable, while its computational cost is acceptable when using single crystal surfaces and model head-groups of up tens of atoms. Its accuracy for adsorption energies has previously been assessed by comparison with accurate experimental adsorption energies and found to be satisfactory when dispersion interactions are properly taken into account [25]. Furthermore, previous tribological studies based on density functional theory (DFT) $[5,26]$ of various lubricant additives on different surfaces obtained results in good agreement with experimental data. The major interaction of the lubricant additives with the surface comes from the adsorption of polar head groups, while the apolar tail ensures solubility and smooth interactions with the base oil to achieve low friction. For this reason, we choose to limit our study to the interactions between the additives head groups and the surface, which is equivalent to consider that the carbon chains of the additives are all the same and play the same role for all additives. The studied polar head groups cover a wide range of additives including protic and aprotic molecules, functionalized aromatics and phosphorus containing compounds. The exhaustive list is given in Table 1 to Table 4.

Regarding the surface model, since aluminium oxidizes immediately when exposed to air, we choose to model the surface by aluminium oxide. Alumina can exhibit different types of crystallographic structures depending on the temperature $[27,28]$. It has been shown that the oxidation of aluminium in air leads to the formation of an amorphous alumina layer of 10 to $20 \AA$ of thickness [29,30]. However, aluminium sheets are fabricated by a rolling process at temperatures between 420 and $530^{\circ} \mathrm{C}$ [31]. Under these conditions, the amorphous alumina layer is transformed into crystalline $\gamma$-alumina [29]. Therefore, $\gamma$ - 
$\mathrm{Al}_{2} \mathrm{O}_{3}$ has been chosen in order to model the oxide layer present at the top of the studied aluminium sheets.

After defining the surface of $\gamma$-alumina which is the most suitable to model the considered system, we will discuss the values of adsorption energies obtained for the different studied head groups of additives. Then, we will study the solvation energies obtained for the same molecules. And finally, we will be able to compare the adsorption and solvation energies in order to assess which types of additives seem to be the most appropriate to improve the wettability of the lubricant on the considered surface.

\section{Models and method}

\subsection{Computational details}

All DFT based computations are carried out using CP2K [32]. The exchange correlation energies were computed with the generalized gradient approximation of Perdew, Burke and Ernzerhof (PBE) [33] with the DFT-D3 dispersion correction developed by Grimme et al. including C9 terms [34,35]. A mixed Gaussian and plane waves (GPW) $[36,37]$ approach has been adopted. A double- $\zeta$ basis set as provided by the Molopt basis set library is used to describe valence electrons, while core electrons were treated with the Goedecker, Teter and Hutter (GTH) pseudopotentials [38-41]. A 400 Ry cutoff energy has been chosen for the plane waves basis set. During the self-consistent field process, the energies are converged to $1 \cdot 10^{-6} \mathrm{Ha}$.

The geometries are optimized at the $\Gamma$-point to forces below $4.5 \cdot 10^{-4} \mathrm{Ha} / \mathrm{bohr}$. The total energies are

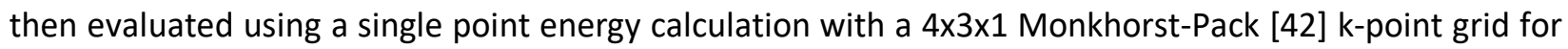
a $p(1 \times 1)$ unit cell and adapted to keep the k-point density constant for larger cells. 
The $\gamma$-alumina (100) surface is modeled by a periodic slab, with a finite thickness to limit the overall computational cost. This slab is non-symmetric, with one of the exposed facet being frozen in the alumina bulk position to mimic the greater thickness of the alumina found on the aluminium sheet, while the opposite facet is free to adapt upon adsorption of any molecules, to mimic the exposed surface of the aluminium sheet. In our case, we chose a $p(1 \times 1), p(2 \times 1)$ or $p(2 \times 2)$ unit cell depending on the size of the adsorbed molecule, with a thickness of 14 atomic planes (two layers), the bottom 8 of which were kept frozen (Figure 1b). A vacuum thickness of $20 \AA$ was set between two periodically repeated slabs to allow the adsorption of molecules on the upper relaxed side of the slab without interacting with the bottom of the periodic image of this slab. The adsorption energy is defined as:

$E_{\text {ads }}=E_{\text {molecule@ } @ \mathrm{Al}_{2} \mathrm{O}_{3}}-\left(E_{\mathrm{Al}_{2} \mathrm{O}_{3}}+E_{\text {molecule }}\right)$

The solvation energy evaluations are performed with Gaussian09 [43] at the DFT level with the PBE functional and a triple- $\zeta$ basis set Def2-TZVP $[44,45]$, using the Polarizable Continuum Model (PCM). The PCM method used here is the Integral Equation Formalism PCM (IEFPCM) [46,47], which is the default one in Gaussian09. In this method, the solvent is described thanks to macroscopic properties such as its permittivity [48]. The solute is treated by quantum mechanics the same way as in vacuum, and the interactions between the solvent and the solute are added by an interaction potential $\widehat{V}_{R}$. Therefore, the vacuum Hamiltonian $\widehat{H}^{0}$ is replaced by an effective Hamiltonian $\widehat{H}^{\text {eff }}$ given by: $\widehat{H}^{\text {eff }}=\widehat{H}^{0}+\widehat{V}_{R}$ [46].

\section{$2.2 \gamma$-alumina}

$\gamma$-alumina is a commonly used material, especially as a catalytic support for heterogeneous catalysts, explaining the abundance of theoretical and experimental studies involving $\gamma$-alumina $[49,50]$. Nevertheless, since it is not perfectly crystalline in reality, its structure is not well known and different 
types of models can be found in the literature [51-53]. In agreement with our previous studies [49,54], we chose to work with the non-spinel model of $\gamma$-alumina proposed by Krokidis et al. [51,55] .

$\gamma$-alumina mainly exposes three different facets: (100), (110) and (111). The (100) surface is the one having the lowest surface energy [55], and therefore the most probably exposed, and has for this reason been adopted in this study.

The $p(1 \times 1)$ unit cell of this surface exhibits four aluminium Alv atoms (Figure 1a), where the subscript $V$ indicates that the atom is coordinated to five oxygen atoms. Two of the aluminium atoms are equivalent $\left(A l_{v b}\right.$ and $\left.A l_{v^{\prime}}\right)$. Three non-equivalent oxygen atoms can also be seen on the surface, one of them being coordinated to four aluminium atoms $\left(\mathrm{O}_{4}\right.$ which is equivalent to $\left.\mathrm{O}_{4^{\prime}}\right)$, whereas the others $\left(\mathrm{O}_{3 a}, \mathrm{O}_{3 a^{\prime}}, \mathrm{O}_{3 b}\right.$ and $\mathrm{O}_{3 b^{\prime}}$ ) are coordinated to three aluminium atoms only.

(a)

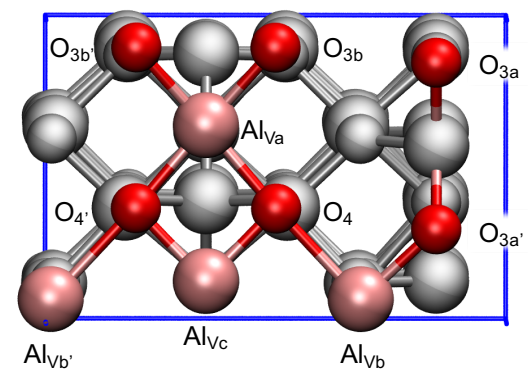

(b)

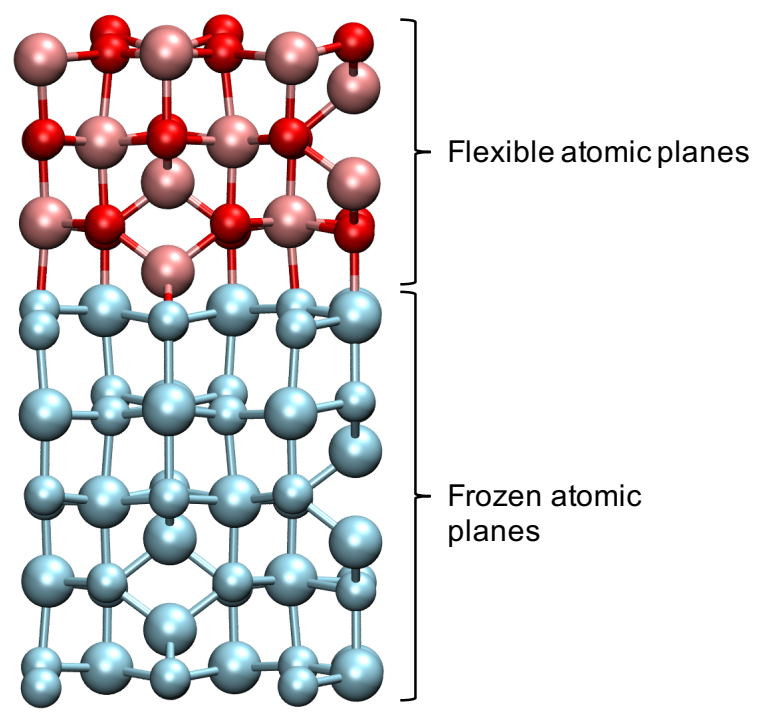

Figure 1 - (a) Top view of the first atomic layer of the $p(1 \times 1)$ unit cell of the (100) surface and the corresponding labeling of the exposed adsorption sites. The other atomic layers are shaded. (b) Side view of the $p(1 \times 1)(100) \gamma$-alumina slab. 


\subsection{Set of studied molecules}

Different kinds of molecules of interest have been studied. These molecules were chosen because they model the polar head group of various additives of fully formulated lubricants. These functional groups thus probe the major adsorption energy contributions of the additives. The chosen molecules have been classified into four categories depending on their characteristics.

Molecules derived from fatty acids containing a polar group, such as a carboxylic acid, alcohols or esters, are often used as friction modifiers in lubricant formulations, whereas amines exhibit good antioxidant and corrosion inhibitor properties [2]. We have, however, chosen to classify them according to basic physical chemical properties. Hence, the two first families gather together respectively protic (Table 1 ) and aprotic (Table 2) small molecules with various functional groups. The third group (Table 3 ) is composed of aromatic molecules. This category serves to determine the influence of the aromatic ring on functional groups of particular interest. Indeed, the steric hindrance induced by the presence of an aromatic group can modify the adsorption of a molecule. The last category gathers phosphorous compounds (Table 4). As they can be used as anti-wear, extreme pressure additives or antioxidants, these compounds are key lubricant additives [2].

Table 1 - Chemical structures of studied protic molecules. Tertiary amine is here classified as a protic molecule due to its similarity

$$
\text { with the protic amines. }
$$

\begin{tabular}{|c|c|}
\hline Name & Structure \\
\hline
\end{tabular}

\begin{tabular}{|c|c|}
\hline Water & Methanol \\
\hline Polyalkylene glycol & \\
\hline
\end{tabular}




Secondary amine

Table 2 - Chemical structures of studied aprotic molecules

Ester (methyl Structure

Table 3 - Chemical structures of studied aromatic molecules

Name Structure




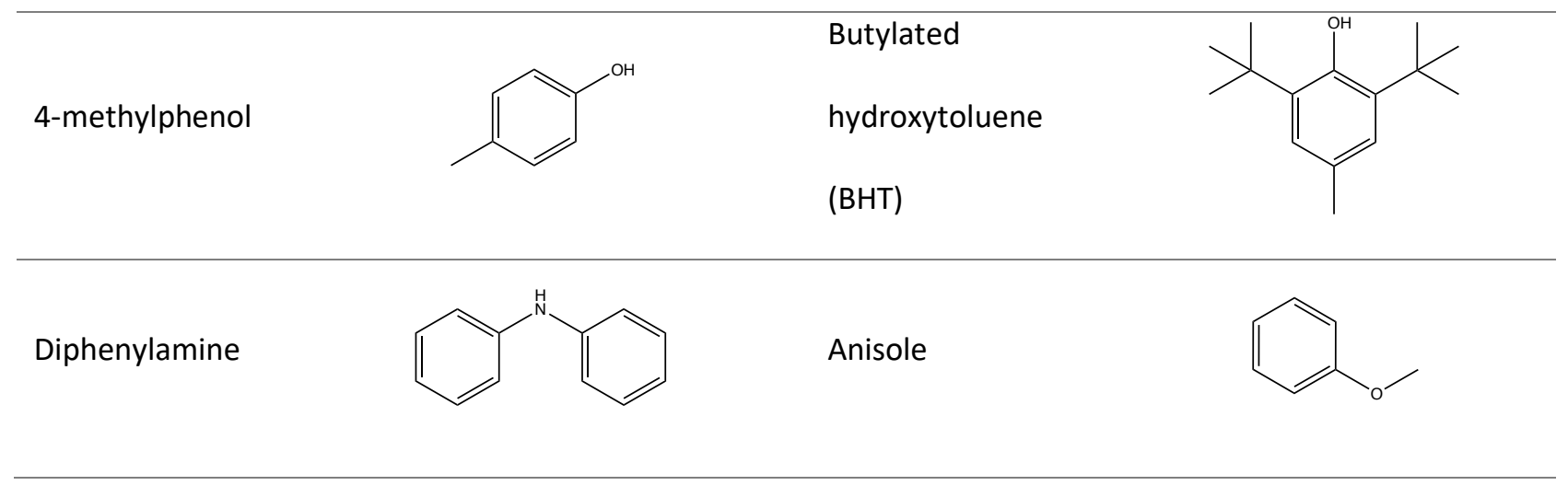

Table 4 - Chemical structures of studied phosphorus-containing molecules

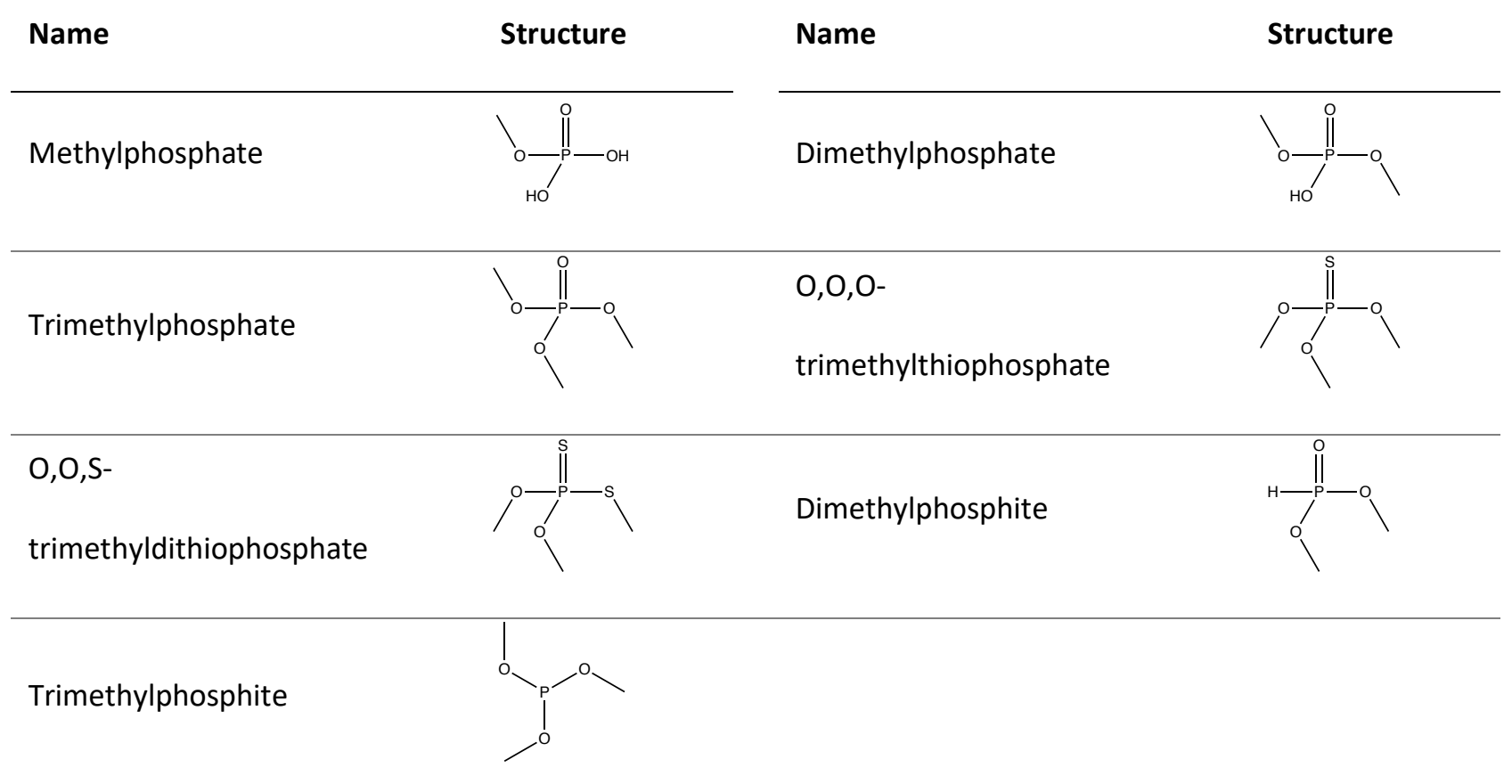

\section{Results and discussion}

\subsection{Adsorption}

Adsorption of molecules on the (100) surface of $\gamma$-alumina is driven by acid/base characteristics of the molecule and surface site, as the aluminium atoms of $\gamma$-alumina are hard Lewis-acidic adsorption sites. 
Both our study and literature [55-57] identified the Alva aluminium atom as the most reactive one, and therefore as the preferential adsorption site. This could have been predicted from the Lewis acidities of the different aluminium atoms of the surface [55]. Indeed, Digne et al. have shown that Alva is the most acidic aluminium atom of the surface. Additionally, surface oxygen atoms can also be involved in the adsorption process, acting as Bronsted basic sites. The adsorption energy of each molecule is reported in Table S1.

\subsubsection{Protic molecules}

For protic molecules, the Bronsted acidity of the molecule is a key parameter to understand the strength of the adsorption.

The water molecule is adsorbed on the (100) $\gamma$-alumina surface with an adsorption energy of $-0.97 \mathrm{eV}$. This adsorption is dissociative: the oxygen atom of the water molecule is adsorbed on the Alva aluminium atom and the hydrogen atom is adsorbed on the $\mathrm{O}_{3 \mathrm{~b}}$ oxygen atom of the surface (Figure $2 \mathrm{a}$ ). This is consistent with previous studies by Digne et al. which obtained the same adsorption mode with a similar energy of $-1.08 \mathrm{eV}[55,56]$. The slight difference in energy observed between this study and the literature can be explained by variations in the computational method.

Given the adsorption mode of water, one could think that the adsorption of methanol would occur analogously. However, as previously published by Zuo et al. [57], we find that the adsorption of methanol is following a non-dissociative mode. This can be rationalized by the fact that methanol has a higher $\mathrm{pK}_{\mathrm{a}}(15.2)$ than water. The adsorption mode corresponds to the oxygen atom of the alcohol adsorbed on $\mathrm{Al}_{\mathrm{Va}}$ with an energy of $-1.07 \mathrm{eV}$. This adsorption energy is slightly stronger than the one 
obtained by Zuo et al. $(-0.88 \mathrm{eV})$ [57], because we account for dispersion interactions. With $-1.00 \mathrm{eV}$, the dissociative adsorption of methanol is nevertheless not significantly less favorable than the nondissociative one. Similarly, methanethiol is adsorbed in a non-dissociative way. Despite larger dispersion interactions, methanethiol is even $0.1 \mathrm{eV}$ less strongly adsorbed than water $(-0.89 \mathrm{eV})$. The selected PAG monomer on the contrary, is adsorbed the same way as water in a dissociative mode on $\mathrm{Al}_{\mathrm{va}}$ and $\mathrm{O}_{3 \mathrm{~b}}$ and sticks more to the surface $(-1.29 \mathrm{eV})$ because of dispersion interactions.

Let us now move to the family of amines, which interact with the $\gamma$-alumina surface through their nitrogen atom. $\mathrm{NH}_{3}$ is not dissociated during the adsorption, but nevertheless reaches an adsorption energy of $-1.24 \mathrm{eV}$. The non-dissociative character of this adsorption was expected because of the high $\mathrm{pK}_{\mathrm{a}}$ value of ammonia, which is equal to 23 . The nitrogen atom of the molecule is still adsorbed on the Alva aluminium atom. The same occurs for primary, secondary and tertiary amines. The adsorption is stronger for the primary amine than for $\mathrm{NH}_{3}$ and even more for the secondary amine (-1.43 and $-1.55 \mathrm{eV}$ respectively). However, the adsorption energy of the tertiary amine $(-1.29 \mathrm{eV})$ is decreasing due to the steric effects. This steric hindrance is also reflected in the geometry: the bond between the nitrogen atom of the tertiary amine and $A \mathrm{~V}_{\mathrm{Va}}$ is much longer $(2.21 \AA)$ than the one observed with primary or secondary amine $(2.05 \AA)$.

Now, let us consider the more complex case of ethoxylated amine. This polydentate molecule preferentially adsorbs in a bidentate mode, involving either the nitrogen and a hydroxyl group, or the two hydroxyl groups (Figure $2 \mathrm{~b}$ and Figure $2 \mathrm{c})$. In the most stable one $(-1.78 \mathrm{eV})$, the $\mathrm{N}$ is not involved: the oxygen atom of the first hydroxyl group and the hydrogen atom of the second one interact with Alva and $\mathrm{O}_{3 a}$, respectively (Figure $2 \mathrm{~b}$ ). This is quite surprising as the adsorption of a tertiary amine is stronger as the one of an alcohol. But, the strongest adsorption obtained by bonding the nitrogen atom to the 
surface has an energy of $-1.41 \mathrm{eV}$, demonstrating that the adsorption of complex multidentate molecules on this alumina surface cannot simply be derived from their sub-groups. The corresponding bi-dentate structure is shown in Figure 2c.

The carboxylic acid also shows a bidentate adsorption, its two oxygen atoms adsorbed on the $\mathrm{Al}_{\mathrm{va}}$ and Alvc sites (Figure $2 \mathrm{~d}$ ). Moreover, the $\mathrm{OH}$ bond is dissociated and the hydrogen atom is adsorbed on the $\mathrm{O}_{3 \mathrm{~b}^{\prime}}$ atom of the surface. This adsorption is very favorable, having an adsorption energy of $-1.96 \mathrm{eV}$. Similarly, two nitrogen atoms of triazole are adsorbed on $\mathrm{Al}_{\mathrm{va}}$ and $\mathrm{A} \mathrm{I}_{\mathrm{vc}}$ and the dissociated hydrogen atom on $\mathrm{O}_{3 b}$, yielding an adsorption energy of $-1.55 \mathrm{eV}$.

From these results, we can conclude that the adsorption of protic molecules on the (100) $\gamma$-alumina surface leads in most cases to a dissociative adsorption for acidic molecules with energies up to $-1.96 \mathrm{eV}$ and that soft Lewis bases such as thiols only interact weakly with the surface. 
(a)
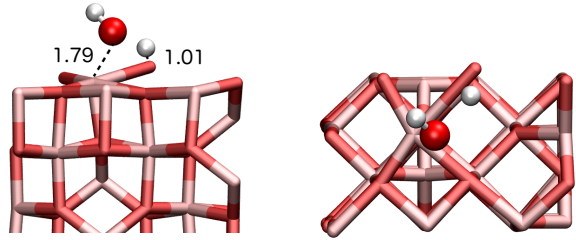

(b)
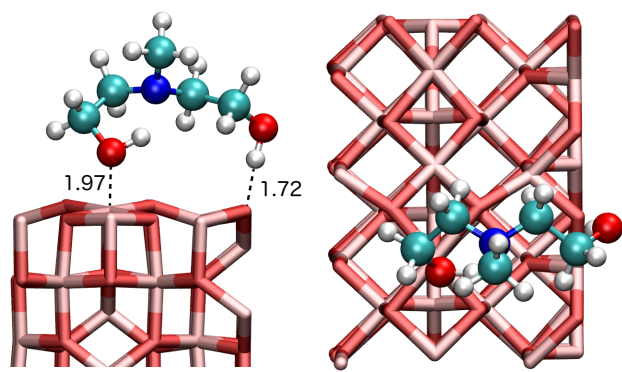

(c)
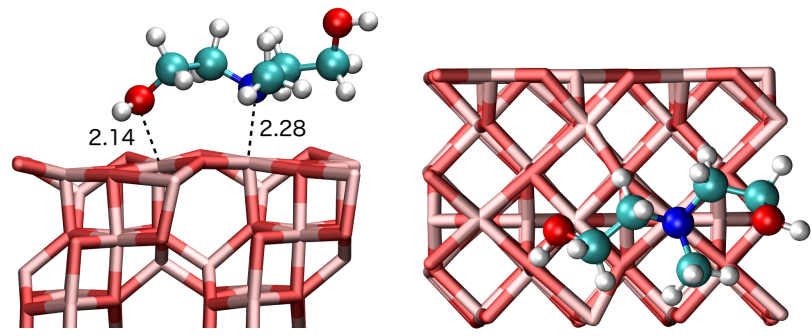

(d)
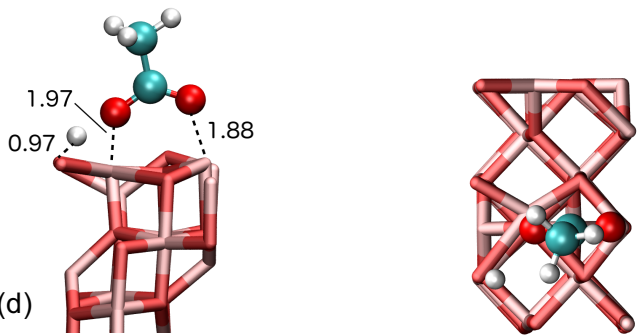

Figure 2 - Adsorption of some selected protic molecules on the (100) surface of $\gamma$-alumina: (a) water, (b) ethoxylated amine, (c) ethoxylated amine with the nitrogen atom adsorbed and (d) carboxylic acid. Distances are given in $\AA$.

As explained previously, some of the studied protic molecules are exhibiting dissociative adsorptions.

This also occurs for some aromatic and phosphorous compounds which are also protic but will be discussed in the following sections. For these molecules, the adsorption energy strongly correlates with the $\mathrm{pK}_{\mathrm{a}}$ value in water (Figure 3 ). The $\mathrm{pK}_{\mathrm{a}}$ indicates the strength of an acid: the lower the value of $\mathrm{pK}_{\mathrm{a}}$, the strongest the acid is. Therefore, the lower the $\mathrm{pK}_{\mathrm{a}}$ value, the more the molecule will have the tendency to dissociate and the stronger the adsorption energy will be. 


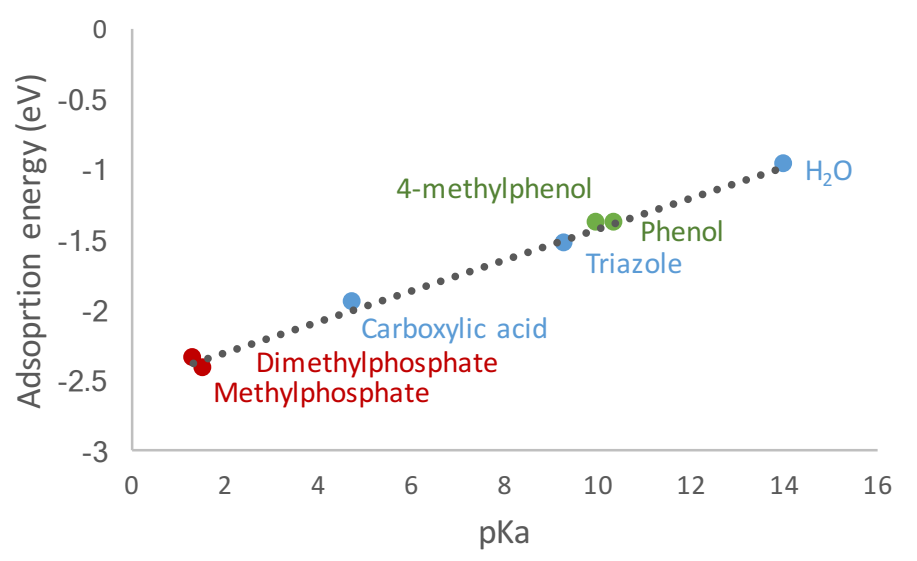

Figure 3 - Correlation of adsorption energy with $p K_{a}$ for molecules exhibiting a dissociative adsorption.

\subsubsection{Aprotic molecules}

The aprotic molecules we selected for this study are Lewis bases and will therefore interact with their electron pair on Alva. The majority of the aprotic molecules under consideration are adsorbed in a monodentate way, but some of them show a bidentate adsorption on the alumina surface.

The adsorption of dimethylether occurs via its oxygen atom and features an energy of $-1.15 \mathrm{eV}$. This is consistent with a previous study [57] that obtained an adsorption energy of $-0.87 \mathrm{eV}$ on the (100) surface of $\gamma$-alumina, with the energy difference coming mainly from the dispersion contribution. The carbonyl oxygen of acetone is a weaker Lewis-base and the interaction energy is indeed slightly weaker $(-1.00 \mathrm{eV})$ compared to the ether. For the ester, either the carbonyl or the other oxygen atom could be the anchoring site. Considering the adsorption of ketone and ether, one could first think that the adsorption of ester would occur on the ether-like oxygen. However, the carbonyl oxygen benefits from electron delocalization in minor Lewis structures and thus becomes the most basic oxygen atom. Hence, the adsorption via the carbonyl oxygen atom reaches $-1.08 \mathrm{eV}$, while the other one leads to only $-0.84 \mathrm{eV}$. 
Dimethylsulfide is adsorbed exactly the same way as the ether but slightly more strongly: the sulfur atom adsorbs on $\mathrm{Al}_{\mathrm{Va}}$ yielding an adsorption energy of $-1.28 \mathrm{eV}$. The greater strength of adsorption results from the combination of a lower destabilizing deformation of the surface with greater stabilizing dispersion interactions.

As the nitrogen atom of amides is not basic, the adsorption of $\mathrm{N}, \mathrm{N}$-dimethylacetamide is analogous to the one of ester and occurs via the oxygen of the carbonyl group. It leads to a relatively strong adsorption energy of $-1.40 \mathrm{eV}$ presumably due to a more important delocalization of electrons from the nitrogen to the carbonyl oxygen, which is also responsible for the more rigid planarity of amide bonds compared to esters [58].

1-methyltriazole adsorbs the same way than triazole, the two nitrogen atoms not linked to the methyl group are adsorbed on the $A I_{v a}$ and $A l_{v c}$ atoms of the surface, the only difference being the fact that 1methyltriazole does not dissociate as it is aprotic. For this reason, its adsorption is weaker than the one observed for triazole as it has an adsorption energy of $-1.19 \mathrm{eV}$, compared to $-1.55 \mathrm{eV}$ for the triazole. For the 1-methyl-1,2,4-triazole, contrary to what has been observed for the triazole and 1-methyltriazole molecules, the bidentate adsorption is not stable. Indeed, while performing a geometry optimization calculation with an initial bidentate adsorption of the molecule on the surface, the molecule moves away from the surface and adsorbs then again in a monodentate way. This can be expected as it is not favorable to adsorb the methylated nitrogen due to steric effects, and the two other nitrogen atoms are too far from each other to allow a bidentate adsorption. Thus, the most favorable geometry is obtained for a monodentate adsorption of the $\mathrm{N}_{4}$ nitrogen atom on Alva with an energy of $-1.44 \mathrm{eV}$. The 1,2dimethyl-4,5-dihydroimidazole molecule is adsorbed on the surface by the less substituted nitrogen 
atom. This can easily be predicted considering steric effects. The resulting adsorption energy $(-1.40 \mathrm{eV})$ is quite strong.

From the results obtained it can be seen that the aprotic molecules studied here exhibit a smaller range of adsorption energies than the one observed with protic molecules and that the adsorption mode is driven by Lewis acid/base pairing.

\subsubsection{Aromatic molecules}

Aromatic groups are present in many lubricant additives. Therefore, it was essential to determine the influence of aromatic rings on functional groups of particular interest.

Benzene is the simplest aromatic compound and it is the common structure of all aromatics tested here. But this molecule does not chemisorb on the (100) $\gamma$-alumina surface and stays flat above the surface, at a distance of about $3 \AA$, which is characteristic of a physisorption. On the contrary, all other aromatic molecules studied here adsorb on the surface, thanks to their substituents.

The adsorption of phenol occurs in analogy to the dissociative adsorption of methanol: its $\mathrm{OH}$ group dissociates and the oxygen atom is adsorbed on the $A \mathrm{l}_{\mathrm{va}}$ aluminium as the hydrogen atom is transferred to the $\mathrm{O}_{3 b^{\prime}}$ oxygen atom. The adsorption of phenol is, however, stronger $(-1.38 \mathrm{eV})$ than the one obtained for methanol. This can be explained by the fact that the dispersion contribution to adsorption energy is greater for the phenol than for the methanol (-0.54 and $-0.27 \mathrm{eV}$ respectively). Moreover, phenol has a higher tendency to dissociate than methanol due to its lower $\mathrm{pK}_{\mathrm{a}}$ value (10.0 for phenol compared to 15.2 for methanol), which explains the difference in dissociation behavior compared to methanol. The 
same occurs for 4-methylphenol, with exactly the same energy than the one observed for the phenol. Butylated hydroxytoluene, on the contrary, does not dissociate nor adsorb on the surface. Indeed, as benzene, it remains at a distance of $3.5 \AA$ above the surface, due to the steric effects induced by the tertbutyl groups.

The adsorption mode of diphenylamine is equivalent to the one of the secondary amine: the nitrogen atom adsorbs on Alva, with an energy of $-1.28 \mathrm{eV}$. The adsorption energy is however weaker, which can be rationalized by the combined effect of electron delocalization of the nitrogen lone-pair with the aromatic cycle and the larger steric hinderance of the phenyl groups compared to the methyl groups. Similarly, the adsorption of anisole follows the one of ether, but is slightly weaker (-1.02 eV).

In a nutshell, the adsorption of aromatic molecules on alumina is driven by the nature of the substituent. When protic, it is driven by the pKa if the substituent can sterically access to the surface. When exhibiting Lewis base properties, it will more or less strongly bind, in a subtle compromise between the Lewis acid/base interaction, the dispersion, steric hindrance, etc.

\subsubsection{Phosphorus-containing molecules}

Phosphorous compounds exhibit a wide range of adsorption energies going from -0.70 to $-2.41 \mathrm{eV}$. They globally have higher adsorption energies than the other molecules studied here, as ether, ketone or alcohol for example. This is due to the fact that phosphorus is less electronegative than carbon, so the $\mathrm{P}-\mathrm{O}$ bond is more polarized than the $\mathrm{C}-\mathrm{O}$ bond and, for this reason, the oxygen atoms of phosphorous compounds are stronger Lewis bases. 
Methylphosphate and dimethylphosphate are adsorbed on the surface alike (Figure 4a). For each of them, one $\mathrm{OH}$ group dissociates, creating two equivalent oxygen atoms on the molecule. These two oxygen atoms are adsorbed on the Al $\mathrm{Va}_{\mathrm{v}}$ and $\mathrm{Al} \mathrm{vc}_{\mathrm{c}}$ atoms of the surface, while the dissociated hydrogen binds to the $\mathrm{O}_{3 b^{\prime}}$ oxygen atom. As a result, this bidentate adsorption mode is very stable, with an adsorption energy of $-2.41 \mathrm{eV}$ and $-2.34 \mathrm{eV}$ for methylphosphate and dimethylphosphate respectively, which is very high compared to all other molecules studied here. The strength of the adsorption lies not only in the formation of two Al-O bonds but also in the low $\mathrm{pK}_{\mathrm{a}}$ values $(1.54$ and 1.29 for methylphosphate and dimethylphosphate respectively), which yields to a dissociative adsorption. Comparatively, as there is no hydroxyl group in the trimethylphosphate molecule, its adsorption is weaker $(-1.61 \mathrm{eV})$ and occurs differently: only its non-substituted oxygen is strongly interacting with $\mathrm{Al}_{\mathrm{Va}}$.

0,0,0-trimethylthiophosphate is very similar to trimethylphosphate, the only difference is the replacement of the non-methylated oxygen by a sulfur atom. For this reason, one could think that the two molecules adsorb in a similar way through the non-substituted atom, sulfur and oxygen, respectively. This adsorption mode is found to lie at $-0.95 \mathrm{eV}$ for the $0,0,0$-trimethylthiophosphate, much weaker than for the fully oxygenated analog $(-1.61 \mathrm{eV})$. However, this is not the most favorable adsorption for the sulfurized derivative. When one of the oxygen atoms is adsorbed on the Alva aluminium atom and a second one on $\mathrm{Al}_{\mathrm{vc}}$ (Figure 4b), an adsorption energy of $-1.10 \mathrm{eV}$ is reached. Then, the adsorption of 0,0,0-trimethylthiophosphate resembles much more the ether adsorption than the other phosphorous compounds as the adsorption is here performed via two ether-like oxygen atoms.

Going further in the sulfurization, let us now consider O,O,S-trimethyldithiophosphate. It presents a bidentate adsorption of $-1.23 \mathrm{eV}$ of the methylated sulfur and of one of the oxygen atom on the $\mathrm{Al}_{\mathrm{Va}}$ and Alvc atoms respectively (Figure 4c). Indeed, despite the fact that this molecule is quite similar to $0,0,0$ - 
trimethylthiophosphate as the only difference is an oxygen atom replaced by a sulfur, they exhibit different adsorptions. This can be intuited regarding the difference of adsorption strength of ether and dimethylsulfide: the latter is more strongly adsorbed than ether, which explains the fact that in the case of 0,O,S-trimethyldithiophosphate, the methylated sulfur sticks to the surface instead of the methylated oxygen.

In summary, since several adsorption modes are in competition for these compounds, the best mode of adsorption cannot be easily predicted and mixtures are likely relevant under experimental conditions.

The adsorption of dimethylphosphite is similar to the one of trimethylphosphate discussed before, i.e., it is the non-substituted oxygen that sticks to the Alva aluminium atom of the surface with an energy of $-1.53 \mathrm{eV}$, slightly lower than the one observed for trimethylphosphate. The trimethylphosphite molecule is quite different from all other phosphorous compounds studied. This molecule shows a bidentate adsorption with two oxygen atoms sticking to the Alva and Alvc aluminium atoms of the surface (Figure $4 \mathrm{~d}$ ) by total adsorption energy of $-0.70 \mathrm{eV}$, which is very low, compared to the other molecules. 
(a)
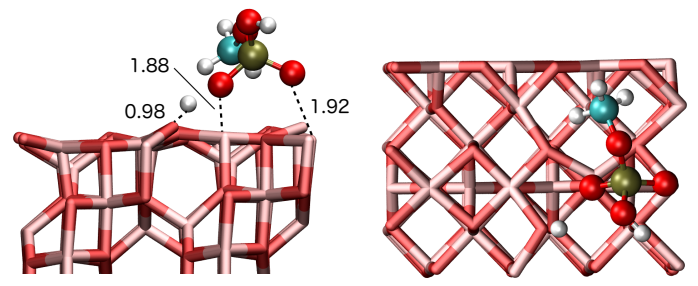

(b)

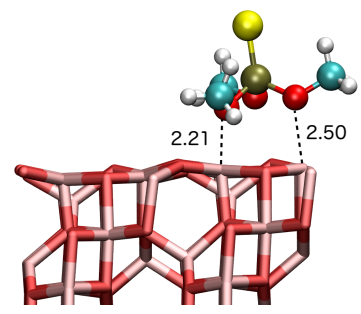

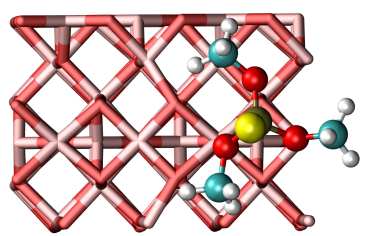

(c)
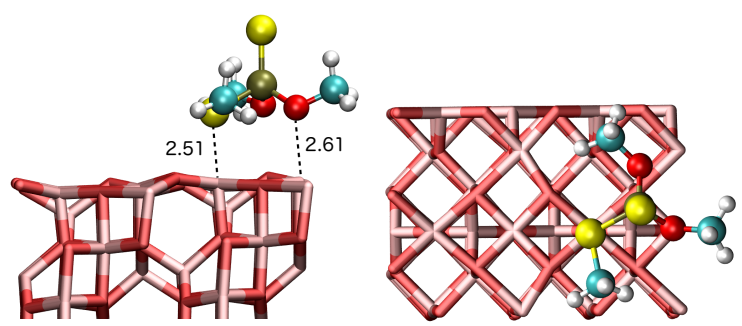

(d)

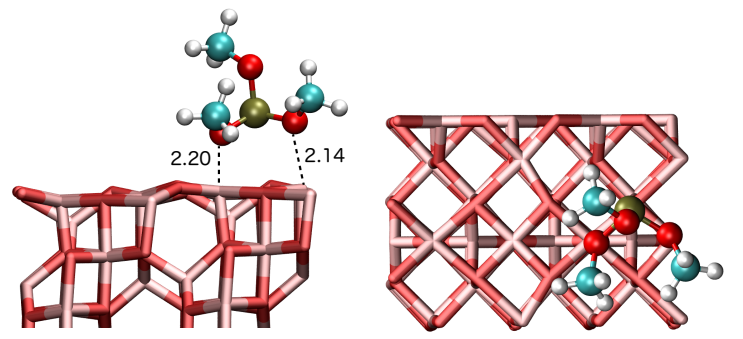

Figure 4 - Adsorption of some selected phosphorous compounds on the (100) surface of $\gamma$-alumina: (a) methylphosphate, (b) 0,0,0-trimethylthiophosphate, (c) 0,0,S-trimethyldithiophosphate and (d) trimethylphosphite. Distances are given in $\AA$.

\subsection{Solvation}

Prediction of solvation effects at the solid/liquid interface is very challenging due to the quasi-absence of reliable experimental reference data. Hence, the use of implicit solvent models [59] or including a few explicit solvent molecules [60] to capture the main influence of the solvent is a common strategy [61,62]. Alternatively, molecular mechanics based simulations also allow to assess the solvation energy at the interface [63], with the issue of identifying a force field which is accurate for the solid liquid interface [64]. Since the base oils are rather apolar, we use here an implicit model to compute solvation energies (see 2.1 for a complete description). This approach was shown to deliver accurate values [65]. We herein make also a drastic approximation: the bulk solution solvation energies are assumed to be a reliable proxy to study solvation effects on the adsorption energy, implicitly assuming that the entire solvation energy is lost upon adsorption. 
The solvation energies of the different molecules studied were calculated in two different solvents: $\mathrm{n}$ hexadecane and pentyl ethanoate. These two solvents were chosen to model two different kinds of base oil used in lubricant formulations: saturated hydrocarbons (group II; modeled by hexadecane) and esters (modeled by pentyl ethanoate). Comparing these two solvents, $\mathrm{n}$-hexadecane has the least solvating power, but trends between different molecules are the same for the two chosen solvents. Quantitatively, the solvation energies in pentyl ethanoate are $81 \%$ higher compared to $\mathrm{n}$-hexadecane. For this reason and as group II base oil are more commonly used in lubricant formulation than ester base oils such as ethylhexyl palmitate, only the solvation in hexadecane will be discussed here.

Since we are assessing the solvation energy of small polar head groups in an apolar solvent, the solvation energies are quite small. Furthermore, they are quite similar, ranging from -0.02 for the tertiary amine to $-0.15 \mathrm{eV}$ for methylphosphate. General tendencies can nevertheless be seen from these results. Aromatic compounds are less solvated than the other types of molecules, with solvation energies of about $-0.07 \mathrm{eV}$. The phosphorous compounds, on the contrary, are on average more solvated than the other molecules. In general, the more substituted a molecule is, the less it is solvated. This phenomenon can be observed for different families of molecules studied here, among which we can find the phosphates and the amines, but also the phosphites, the triazoles... As an example, let us consider the phosphates which have quite strong solvation energies. Methylphosphate is better solvated $(-0.15 \mathrm{eV})$ than dimethylphosphate $(-0.13 \mathrm{eV})$ while trimethylphosphate is even less solvated $(-0.10 \mathrm{eV})$. For the amines, the primary amine has a solvation energy of $-0.05 \mathrm{eV}$ which is weaker than the one of ammonia $(-0.06 \mathrm{eV})$ but stronger than the one calculated for the secondary amine $(-0.03 \mathrm{eV})$ which is also stronger than the energy obtained for the solvation of the tertiary amine $(-0.02 \mathrm{eV})$. 
Even more generally, the results show that more polar molecules are better solvated. This result can be quite surprising on first sight given that we are discussing a non-polar solvent. However, it can be explained by the fact that we are looking only at the solvation energy and not considering the problem of miscibility. Indeed, if we would like to assess the miscibility (or solubility), two different phenomena have to be considered. The first one is that polar molecules are always more solvated than non-polar ones, independently of the characteristics of the solvent. The second phenomenon is the competition between molecule-molecule and molecule-solvent interactions. In apolar solvents, molecule-molecule interactions are more favorable for polar molecules than molecule-solvent interactions, which is the reason for demixing in apolar solvents. Demixing and low solubility is, however, not a real issue in our case since the real additives contain a long hydrophobic tail that ensures solubility in the apolar base oil and we assume that this tail will influence all head groups in roughly the same way.

\subsection{Comparison between adsorption and solvation energies}

After having studied separately both the adsorption and the solvation energies of the different polar head groups of additives, we now compare the adsorption with the solvation energies. As solid-liquid interactions are favoring the wetting of the surface [22], we are looking for additives which have the tendency to go at the interface between the solid surface and the lubricant rather than to stay in the bulk liquid, in order to get better wettability properties, i.e. a better spreading of the lubricant over the surface. For this reason, additives with a high adsorption energy and a low solvation energy are the most promising ones.

For each family of molecules, the adsorption and the solvation energies of the different studied molecules are plotted in Figure 5. 

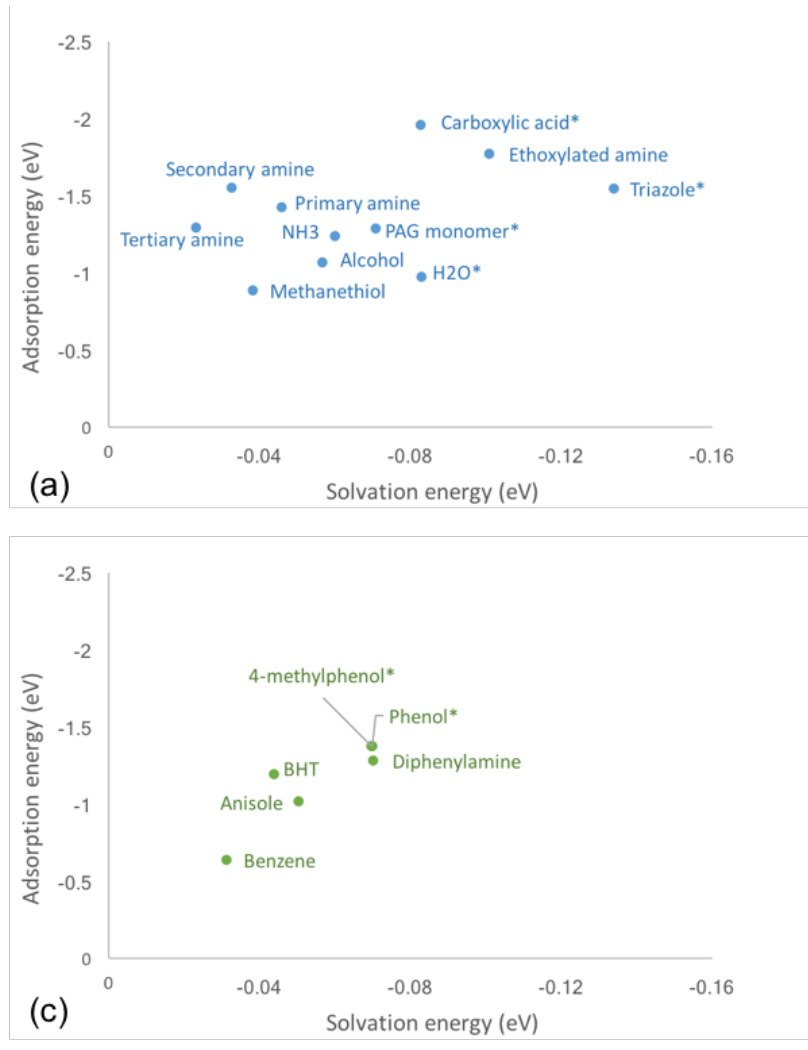
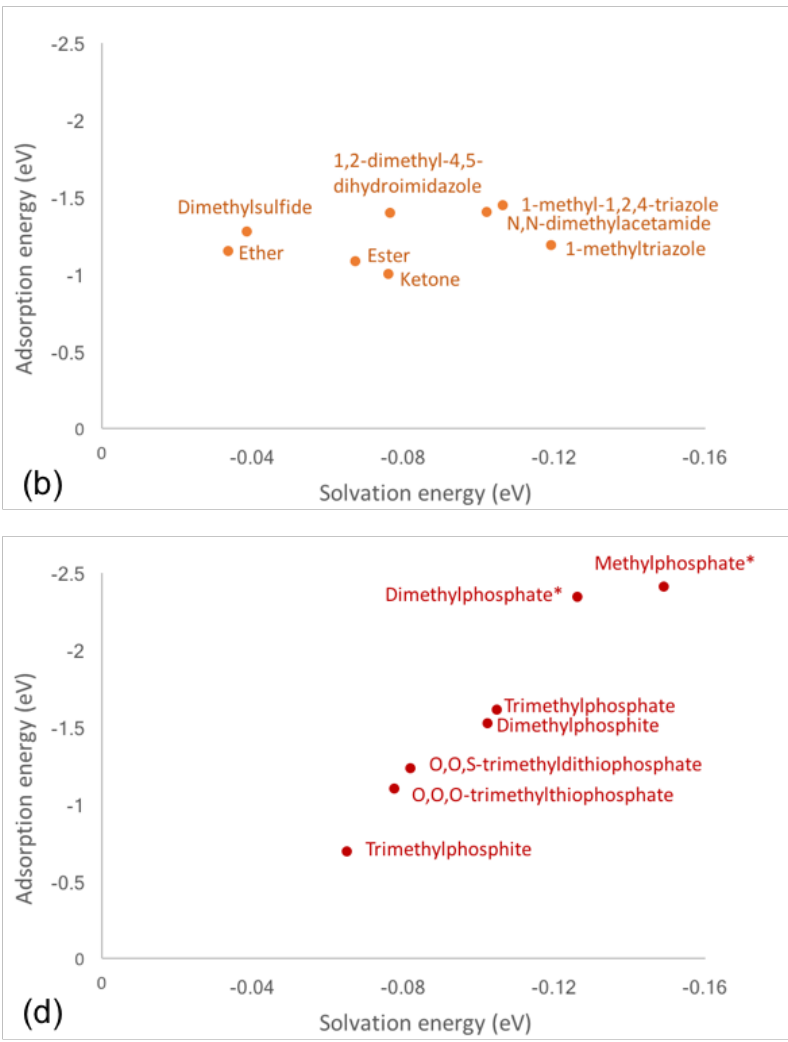

Figure 5 - Adsorption energy as a function of solvation energy for the studied additives: (a) protic molecules, (b) aprotic molecules, (c) aromatic compounds and (d) phosphorous compounds. * indicates dissociative adsorptions.

On Figure 5a, it can be observed that for the protic molecules the adsorption energies are quite similar and barely correlated with the solvation energies. For aprotic molecules (Figure 5b), the adsorption energies fall in a small range $(-1.00$ to $-1.44 \mathrm{eV})$ and there is no correlation at all between the adsorption and solvation energy. On the contrary, for the aromatic and the phosphorous compounds the adsorption energies are strongly correlated with the solvation energies as all the points are on the same line in Figure $5 c$ and Figure $5 d$ respectively. In other words, for these two families, a stronger surface adsorption is only achieved for molecules that also get better solvated.

Despite the qualitative character of our computations (neglect of entropy, single molecule adsorption, solid-vacuum interface and absence of the hydrophobic tail) compared to lubrication, our analysis 
suggests, for instance, that secondary amines are more likely to improve the wettability of a lubricant than phenols: the adsorption energy is very similar, but the solvation energy of the phenols is slightly higher. Similarly, given their similar adsorption energy, dimethylphosphate seems preferable over methylphosphate, since the latter is more solvated than the former. Moreover, by comparing their adsorption energies, our results suggest that carboxylic acids will improve the wettability properties better than alcohols or the esters, which is in agreement with a previous experimental study by Zhou et al. [8] concluding that acids are improving the dynamic wetting of an oil on an aluminium surface better than alcohols and esters.

\section{Conclusion}

In this study, we have performed DFT computations to compare the solvation and adsorption energies of various head groups of lubricant additives in order to investigate which ones are the most suitable to favor the wetting of an alumina surface. We show that the adsorption energies of the studied molecules cover a wide range going from -0.64 to $-2.41 \mathrm{eV}$. On the contrary, their solvation energies are quite similar and are much smaller in absolute value than the adsorption energies.

Our results show that acidic protic molecules, such as carboxylic acid, tend to be dissociated when adsorbed on the (100) surface of $\gamma$-alumina, whereas dissociations do not occur for non-acidic protic head groups. The behavior of aromatic molecules generally follows from their functional groups, as the aromatic part does not stick on the surface. The studied phosphorous compounds exhibit a wide range of adsorption energies. The phosphates are the most adsorbed head groups, which suggests that the lubricant additives containing phosphate head groups should be better lubricant additives to improve 
the wetting of the aluminium surface by the lubricant than the additives containing the other studied head groups.

This study shows that the behavior of a lubricant additive at the base oil/surface interface can be unraveled by DFT. While the solvation plays only a minor role and is not a discriminating parameter, the adsorption energy appears as a key parameter. We also identified that acidic additives adsorb more strongly on alumina, with a strength that can be correlated with a typical chemical property, the pKa. The stronger the adsorption, the more likely is the formation of a film, which will, eventually, reduce friction and wear at the macroscopic scale. This DFT study can easily be extended to other surfaces of interest and other families of additive to better rationalize the choice of lubricant additives.

\section{Supplementary material}

Supplementary material to this article can be found online at: $x x x$

All optimized structures of adsorbed molecules are available in the xyz format.

\section{Acknowledgements}

S. Blanck gratefully acknowledges Total MS for her PhD fellowship. The authors would like to express their gratitude to the PSMN data center for CPU hours and assistance.

\section{References}

[1] Ayel J. Lubrifiants - Constitution. Tech Ing 1997.

[2] Rudnick LR, editor. Lubricant additives: chemistry and applications. 2nd ed. CRC Press; 2009.

[3] Kajdas C. Additives for metalworking lubricants-a review. Lubr Sci 1989;1:385-409. 
[4] Minami I, Mori S. Concept of molecular design towards additive technology for advanced lubricants. Lubr Sci 2007;19:127-49. https://doi.org/10.1002/ls.37.

[5] Righi MC, Loehlé S, De Barros Bouchet MI, Mambingo-Doumbe S, Martin JM. A comparative study on the functionality of S- and P-based lubricant additives by combined first principles and experimental analysis. RSC Adv 2016;6:47753-60. https://doi.org/10.1039/C6RA07545B.

[6] Lingala S, Jayne D, Ernst F. Effect of lubricant additives on the tribological behavior of aluminum alloy against steel. Int J Mater Res 2018;109:789-802.

[7] Wan Y, Xue Q, Liu W. Tribological behavior of lubricating oil additives in lubricated aluminumon-steel contact. Wear 1996;196:87-91. https://doi.org/10.1016/0043-1648(95)06868-6.

[8] Zhou Y, Zhou H. Dynamic wetting of rolling oil on aluminum surfaces. J Cent South Univ Technol 2007;14:255-9. https://doi.org/10.1007/s11771-007-0051-y.

[9] Arieta FG, Gawne DT. Experimental technique to determine the wettability of surfaces to lubricating oils. J Mater Sci 1986;21:1793-1800.

[10] Vakis AI, Yastrebov VA, Scheibert J, Nicola L, Dini D, Minfray C, et al. Modeling and simulation in tribology across scales: An overview. Tribol Int 2018;125:169-99. https://doi.org/10.1016/j.triboint.2018.02.005.

[11] Nishi T, Yamaguchi T, Shibata K, Hokkirigawa K. Influence of unforced dewetting and enforced wetting on real contact formation and friction behavior between rubber hemisphere and glass plate during contacting and sliding processes. Tribol Int 2020;141:105921. https://doi.org/10.1016/j.triboint.2019.105921.

[12] Wong T-S, Ho C-M. Dependence of Macroscopic Wetting on Nanoscopic Surface Textures. Langmuir 2009;25:12851-4. https://doi.org/10.1021/1a902430w.

[13] Anastasiadis SH. Development of Functional Polymer Surfaces with Controlled Wettability. Langmuir 2013;29:9277-90. https://doi.org/10.1021/1a400533u.

[14] Di Mundo R, Palumbo F, d'Agostino R. Influence of Chemistry on Wetting Dynamics of Nanotextured Hydrophobic Surfaces. Langmuir 2010;26:5196-201. https://doi.org/10.1021/1a903654n. 
[15] Li X-M, Reinhoudt D, Crego-Calama M. What do we need for a superhydrophobic surface? A review on the recent progress in the preparation of superhydrophobic surfaces. Chem Soc Rev 2007;36:1350. https://doi.org/10.1039/b602486f.

[16] Feng XJ, Jiang L. Design and Creation of Superwetting/Antiwetting Surfaces. Adv Mater 2006;18:3063-78. https://doi.org/10.1002/adma.200501961.

[17] Kietzig A-M, Hatzikiriakos SG, Englezos P. Patterned Superhydrophobic Metallic Surfaces. Langmuir 2009;25:4821-7. https://doi.org/10.1021/la8037582.

[18] Kumar A, Gogoi B. Development of durable self-cleaning superhydrophobic coatings for aluminium surfaces via chemical etching method. Tribol Int 2018;122:114-8. https://doi.org/10.1016/j.triboint.2018.02.032.

[19] Yuan Y, Lee TR. Contact Angle and Wetting Properties. In: Bracco G, Holst B, editors. Surf. Sci. Tech., vol. 51, Berlin, Heidelberg: Springer Berlin Heidelberg; 2013, p. 3-34. https://doi.org/10.1007/9783-642-34243-1_1.

[20] Gim S, Lim H-K, Kim H. Multiscale Simulation Method for Quantitative Prediction of Surface Wettability at the Atomistic Level. J Phys Chem Lett 2018;9:1750-8. https://doi.org/10.1021/acs.jpclett.8b00466.

[21] Kalin M, Polajnar M. The correlation between the surface energy, the contact angle and the spreading parameter, and their relevance for the wetting behaviour of DLC with lubricating oils. Tribol Int 2013;66:225-33. https://doi.org/10.1016/j.triboint.2013.05.007.

[22] Blake TD, De Coninck J. The influence of solid-liquid interactions on dynamic wetting. Adv Colloid Interface Sci 2002;96:21-36.

[23] Bertrand E, Blake TD, Coninck JD. Influence of solid-liquid interactions on dynamic wetting: a molecular dynamics study. J Phys Condens Matter 2009;21:464124. https://doi.org/10.1088/0953$8984 / 21 / 46 / 464124$.

[24] Nørskov JK, Bligaard T, Hvolbæk B, Abild-Pedersen F, Chorkendorff I, Christensen CH. The nature of the active site in heterogeneous metal catalysis. Chem Soc Rev 2008;37:2163. 
https://doi.org/10.1039/b800260f.

[25] Gautier S, Steinmann SN, Michel C, Fleurat-Lessard P, Sautet P. Molecular adsorption at Pt(111). How accurate are DFT functionals? Phys Chem Chem Phys 2015;17:28921-30. https://doi.org/10.1039/C5CP04534G.

[26] Gattinoni C, Ewen JP, Dini D. Adsorption of Surfactants on $\alpha-\mathrm{Fe}_{2} \mathrm{O}_{3}$ (0001): A Density Functional Theory Study. J Phys Chem C 2018;122:20817-26. https://doi.org/10.1021/acs.jpcc.8b05899.

[27] Wefers K, Misra C. Oxides and hydroxides of aluminum. Alcoa Laboratories; 1987.

[28] Santos PS, Santos HS, Toledo SP. Standard transition aluminas. Electron microscopy studies. Mater Res 2000;3:104-114.

[29] Jeurgens LPH, Sloof WG, Tichelaar FD, Mittemeijer EJ. Structure and morphology of aluminiumoxide films formed by thermal oxidation of aluminium. Thin Solid Films 2002;418:89-101.

[30] Olefjord I, Nylund A. Surface analysis of oxidized aluminium. 2. Oxidation of aluminium in dry and humid atmosphere studied by ESCA, SEM, SAM and EDX. Surf Interface Anal 1994;21:290-297.

[31] Deneuville P. Mise en forme de l'aluminium - laminage. Tech Ing 2010.

[32] Hutter J, Iannuzzi M, Schiffmann F, VandeVondele J. cp2k: atomistic simulations of condensed matter systems. Wiley Interdiscip Rev Comput Mol Sci 2014;4:15-25. https://doi.org/10.1002/wcms.1159.

[33] Perdew JP, Burke K, Ernzerhof M. Generalized Gradient Approximation Made Simple. Phys Rev Lett 1996;77:3865-8. https://doi.org/10.1103/PhysRevLett.77.3865.

[34] Grimme S, Antony J, Ehrlich S, Krieg H. A consistent and accurate ab initio parametrization of density functional dispersion correction (DFT-D) for the 94 elements $\mathrm{H}-\mathrm{Pu}$. J Chem Phys 2010;132:154104. https://doi.org/10.1063/1.3382344.

[35] Grimme S, Ehrlich S, Goerigk L. Effect of the damping function in dispersion corrected density functional theory. J Comput Chem 2011;32:1456-65. https://doi.org/10.1002/jcc.21759.

[36] Lippert G, Hutter J, Parrinello M. A hybrid Gaussian and plane wave density functional scheme. Mol Phys 1997;92:477-88. https://doi.org/10.1080/002689797170220. 
[37] VandeVondele J, Krack M, Mohamed F, Parrinello M, Chassaing T, Hutter J. Quickstep: Fast and accurate density functional calculations using a mixed Gaussian and plane waves approach. Comput Phys Commun 2005;167:103-28. https://doi.org/10.1016/j.cpc.2004.12.014.

[38] Goedecker S, Teter M, Hutter J. Separable dual-space Gaussian pseudopotentials. Phys Rev B 1996;54:1703-10. https://doi.org/10.1103/PhysRevB.54.1703.

[39] Hartwigsen C, Goedecker S, Hutter J. Relativistic separable dual-space Gaussian pseudopotentials from H to Rn. Phys Rev B 1998;58:3641-62. https://doi.org/10.1103/PhysRevB.58.3641.

[40] Krack M. Pseudopotentials for $\mathrm{H}$ to $\mathrm{Kr}$ optimized for gradient-corrected exchange-correlation functionals. Theor Chem Acc 2005;114:145-52. https://doi.org/10.1007/s00214-005-0655-y.

[41] VandeVondele J, Hutter J. Gaussian basis sets for accurate calculations on molecular systems in gas and condensed phases. J Chem Phys 2007;127:114105. https://doi.org/10.1063/1.2770708.

[42] Monkhorst HJ, Pack JD. Special points for Brillouin-zone integrations. Phys Rev B 1976;13:5188-92. https://doi.org/10.1103/PhysRevB.13.5188.

[43] Frisch MJ, Trucks GW, Schlegel HB, Scuseria GE, Robb MA, Cheeseman JR, et al. Gaussian 09. Gaussian, Inc.; 2009.

[44] Weigend F, Ahlrichs R. Balanced basis sets of split valence, triple zeta valence and quadruple zeta valence quality for H to Rn: Design and assessment of accuracy. Phys Chem Chem Phys 2005;7:3297. https://doi.org/10.1039/b508541a.

[45] Weigend F. Accurate Coulomb-fitting basis sets for $\mathrm{H}$ to Rn. Phys Chem Chem Phys 2006;8:1057. https://doi.org/10.1039/b515623h.

[46] Mennucci B, Cancès E, Tomasi J. Evaluation of Solvent Effects in Isotropic and Anisotropic Dielectrics and in Ionic Solutions with a Unified Integral Equation Method: Theoretical Bases, Computational Implementation, and Numerical Applications. J Phys Chem B 1997;101:10506-17. https://doi.org/10.1021/jp971959k.

[47] Cancès E, Mennucci B, Tomasi J. A new integral equation formalism for the polarizable continuum model: Theoretical background and applications to isotropic and anisotropic dielectrics. J 
Chem Phys 1997;107:3032-41. https://doi.org/10.1063/1.474659.

[48] Tomasi J, Mennucci B, Cammi R. Quantum Mechanical Continuum Solvation Models. Chem Rev 2005;105:2999-3094. https://doi.org/10.1021/cr9904009.

[49] Réocreux R, Jiang T, Iannuzzi M, Michel C, Sautet P. Structuration and Dynamics of Interfacial Liquid Water at Hydrated $\gamma$-Alumina Determined by ab Initio Molecular Simulations: Implications for Nanoparticle Stability. ACS Appl Nano Mater 2018;1:191-9. https://doi.org/10.1021/acsanm.7b00100.

[50] Ngouana-Wakou BF, Cornette P, Corral Valero M, Costa D, Raybaud P. An Atomistic Description of the $\gamma$-Alumina/Water Interface Revealed by Ab Initio Molecular Dynamics. J Phys Chem C 2017;121:10351-63. https://doi.org/10.1021/acs.jpcc.7b00101.

[51] Krokidis X, Raybaud P, Gobichon A-E, Rebours B, Euzen P, Toulhoat H. Theoretical Study of the Dehydration Process of Boehmite to $\gamma$-Alumina. J Phys Chem B 2001;105:5121-30. https://doi.org/10.1021/jp0038310.

[52] Gutiérrez G, Taga A, Johansson B. Theoretical structure determination of $\gamma-\mathrm{Al}_{2} \mathrm{O}_{3}$. Phys Rev B 2001;65. https://doi.org/10.1103/PhysRevB.65.012101.

[53] Ferreira AR, Martins MJF, Konstantinova E, Capaz RB, Souza WF, Chiaro SSX, et al. Direct comparison between two structural models by DFT calculations. J Solid State Chem 2011;184:1105-11. https://doi.org/10.1016/j.jssc.2011.03.016.

[54] Réocreux R, Girel É, Clabaut P, Tuel A, Besson M, Chaumonnot A, et al. Reactivity of shapecontrolled crystals and metadynamics simulations locate the weak spots of alumina in water. Nat Commun 2019;10:3139. https://doi.org/10.1038/s41467-019-10981-9.

[55] Digne M, Sautet P, Raybaud P, Euzen P, Toulhoat H. Use of DFT to achieve a rational understanding of acid-basic properties of $\gamma$-alumina surfaces. J Catal 2004;226:54-68. https://doi.org/10.1016/j.jcat.2004.04.020.

[56] Digne M, Sautet P, Raybaud P, Euzen P, Toulhoat H. Hydroxyl Groups on $\gamma$-Alumina Surfaces: A DFT Study. J Catal 2002;211:1-5. https://doi.org/10.1006/jcat.2002.3741.

[57] Zuo Z-J, Han P-D, Hu J-S, Huang W. Effect of surface hydroxyls on DME and methanol 
adsorption over $\gamma$-A12O3 (hkl) surfaces and solvent effects: a density functional theory study. J Mol Model 2012;18:5107-11. https://doi.org/10.1007/s00894-012-1495-z.

[58] MacArthur MW, Thornton JM. Deviations from Planarity of the Peptide Bond in Peptides and Proteins. J Mol Biol 1996;264:1180-95. https://doi.org/10.1006/jmbi.1996.0705.

[59] Fisicaro G, Genovese L, Andreussi O, Mandal S, Nair NN, Marzari N, et al. Soft-Sphere Continuum Solvation in Electronic-Structure Calculations. J Chem Theory Comput 2017;13:3829-45. https://doi.org/10.1021/acs.jctc.7b00375.

[60] Desai SK, Pallassana V, Neurock M. A Periodic Density Functional Theory Analysis of the Effect of Water Molecules on Deprotonation of Acetic Acid over Pd(111). J Phys Chem B 2001;105:9171-82. https://doi.org/10.1021/jp002797m.

[61] Schweitzer B, Steinmann SN, Michel C. Can microsolvation effects be estimated from vacuum computations? A case-study of alcohol decomposition at the $\mathrm{H}_{2} \mathrm{O} / \mathrm{Pt}(111)$ interface. Phys Chem Chem Phys 2019;21:5368-77. https://doi.org/10.1039/C8CP06331A.

[62] Michel C, Zaffran J, Ruppert AM, Matras-Michalska J, Jędrzejczyk M, Grams J, et al. Role of water in metal catalyst performance for ketone hydrogenation: a joint experimental and theoretical study on levulinic acid conversion into gamma-valerolactone. Chem Commun 2014;50:12450-3. https://doi.org/10.1039/C4CC04401K.

[63] Steinmann SN, Sautet P, Michel C. Solvation free energies for periodic surfaces: comparison of implicit and explicit solvation models. Phys Chem Chem Phys 2016;18:31850-61. https://doi.org/10.1039/C6CP04094B.

[64] Steinmann SN, Ferreira De Morais R, Götz AW, Fleurat-Lessard P, Iannuzzi M, Sautet P, et al. Force Field for Water over Pt(111): Development, Assessment, and Comparison. J Chem Theory Comput 2018;14:3238-51. https://doi.org/10.1021/acs.jctc.7b01177.

[65] Cossi M, Rega N, Scalmani G, Barone V. Energies, structures, and electronic properties of molecules in solution with the C-PCM solvation model. J Comput Chem 2003;24:669-81. https://doi.org/10.1002/jcc.10189. 
